\title{
Interfaces
}

\section{De l'Américanité à la Montréalité : Contours d'expériences photographiques formatrices de Melvin Charney}

\section{Élène Levasseur}

\section{OpenEdition}

Journals

Édition électronique

URL : http://journals.openedition.org/interfaces/1531

DOI : 10.4000/interfaces. 1531

ISSN : 2647-6754

Éditeur :

Université de Bourgogne, Université de Paris, College of the Holy Cross

\section{Édition imprimée}

Date de publication : 15 décembre 2020

Pagination : 53-77

ISSN : 1164-6225

\section{Référence électronique}

Élène Levasseur, « De l'Américanité à la Montréalité : Contours d'expériences photographiques formatrices de Melvin Charney », Interfaces [En ligne], 44 | 2020, mis en ligne le 15 décembre 2020, consulté le 21 décembre 2020. URL : http://journals.openedition.org/interfaces/1531 ; DOI : https:// doi.org/10.4000/interfaces. 1531

\section{(c) (†)}

Les contenus de la revue Interfaces sont mis à disposition selon les termes de la Licence Creative Commons Attribution 4.0 International. 


\title{
DE L'AMÉRICANITÉ À LA MONTRÉALITÉ: CONTOURS D'EXPÉRIENCES PHOTOGRAPHIQUES FORMATRICES DE MELVIN CHARNEY*
}

\author{
Élène Levasseur \\ Université de Montréal, medialabAU
}

\begin{abstract}
Résumé : Architecte, artiste et théoricien montréalais, Melvin Charney (1935-2012) a utilisé la photographie comme un outil pour produire des savoirs sur l'urbain et pour révéler ceux qui sont inscrits dans la matière du bâti vernaculaire. Si ces faits sont déjà bien documentés, les origines de son usage de la photographie comme moyen d'apprendre et de penser l'architecture sont restées durablement méconnues. Le dépouillement de ses archives personnelles et l'analyse de documents qu'il a produits avant et pendant ses études en architecture ont récemment permis de mieux les connaître. En particulier, les recherches entreprises ont permis de découvrir le rôle déterminant des œuvres photographiques d'Erich Mendelsohn, de Walker Evans et d'Edward Ruscha dans la formation de sa pensée. Outre le fait d'offrir un éclairage nouveau sur la généalogie de l'œuvre de Charney, cet article vise à réfléchir à la portée cognitive de la photographie et, plus largement, de l'expérience visuelle comme outil pour penser l'architecture.
\end{abstract}

Mots-clefs : photographie, architecture vernaculaire, environnement bâti, Charney (Melvin), Mendelsohn (Erich), Evans (Walker), Ruscha (Edward), ordinaire

\begin{abstract}
Photography was for Melvin Charney a tool for deepening his approach to the urban environment. This fact is already well documented. However, the origins of his photographic experiences as a tool for learning and thinking architecture remain unknown. The exploration of Charney's personal archives and the analysis of documents produced before and during his studies in architecture have recently led to a better understanding of how photography played a part in his training and his vision as an architect, in particular the role played by the photographic output of Erich Mendelsohn and Walker Evans. In addition to shedding new light on the genealogy of Charney's work, including, for example, his references to the work of Edward Ruscha, this article contributes to thinking about the cognitive scope of photography in architecture.
\end{abstract}

Keywords: photography, vernacular architecture, built environment, Charney (Melvin), Mendelsohn (Erich), Evans (Walker), Ruscha (Edward), ordinary

Cet article comporte des images non libres de droit et pour lesquelles nous n'avons pas obtenu d'autorisation de publication en ligne. Seuls les titres et légendes sont indiquées pour le format électronique. Les illustrations sont présentes dans le format pdf. 
God, so to speak, is to be found in a knowledge of humanity embodied in material traces [...]. Melvin Charney, $1996^{1}$

Les expériences photographiques de l'architecte, artiste et théoricien montréalais Melvin Charney (1935-2012) pendant ses années de formation ont teinté de manière significative sa pensée et son œuvre. Dans le sillage des photographies de l'architecte allemand Erich Mendelsohn (18871953 ) et des photographes étasuniens Walker Evans (1903-1975) et Edward Ruscha (1937-), qui, par leurs investigations de terrain, ont donné à voir certains des traits emblématiques de l'Américanité, Charney a documenté une «architecture authentique $»^{2}$ et a eu pour objectif de réévaluer et réinventer les conventions qui régissent le bâti québécois, jusqu'à définir la Montréalité. Ses écrits, œuvres et expositions des années 1970 sont d'ailleurs d'excellents porte-étendards de cette approche visuelle de l'architecture. La photographie du bâti vernaculaire a été pour lui un moyen de mettre au jour un savoir-faire architectural, d'identifier les formes d'une architecture qu'il a qualifiée d'authentique et, plus largement, ce que nous appellerons des bases honnêtes pour l'histoire de l'architecture contemporaine nord-américaine - honnêtes dans la mesure où elles correspondent à une réalité observable et par opposition aux énoncés dogmatiques de l'architecture moderne ${ }^{3}$. Les images photographiques qu'il a produites et récoltées ont également été sources de réflexion, outils d'analyse architecturale et matériaux de nombreux écrits et productions artistiques où il «met en jeu» ${ }^{4} 1$ 'architecture.

Les écrits et les œuvres de Charney ont déjà été l'objet d'un grand nombre de publications ${ }^{5}$, notamment de la part de l'historien de la photographie David Harris qui s'est intéressé à l'ensemble

1. Phrase tirée d'une transcription d'un discours prononcé en février 1996: CCA / Oxford-Cambridge Lecture. Fonds Melvin Charney, Centre Canadien d'Architecture (CCA), $n^{\circ}$ de dossier: DR2012:0012:103:023.

2. Terme utilisé par Charney (1971 13) pour qualifier une architecture «née dans les choses concrètes et enracinée dans la vie des gens», en opposition avec «l'architecture comme système institutionnalisé».

3. L'honnêteté est le qualificatif employé par Rodtchenko au sujet de l'approche photographique que Mendelsohn a développée aux États-Unis en 1924 (Cohen 234). Il le fut également par l'architecte John M. Johansen dans une lettre de recommandation, datée du 4 mai 1960 et destiné à l'Institut royald'architecture du Canada (IRAC), au sujet de l'approche architecturale que Charney avait adoptée alors qu'il était encore étudiant. Fonds Melvin Charney, CCA, $\mathrm{n}^{\circ} \mathrm{de}$ dossier: DR2012:0012:008:022. Cet écho nous semble important en raison d'une proximité et d'un rapport d'influence (que nous détaillerons plus loin) entre la pratique photographique de Mendelsohn en 1924 et celle de Charney dans les années 1950, participant d'un rapport attentif et anti-institutionnel au bâti existant.

4. Termes utilisés par Charney (1991 26) pour définir sa pratique.

5. Dans l'ouvrage publié par le Musée d'Art Contemporain de Montréal et dirigé par Landry en 2002, figure une sélection de plus de cent publications (livres, monographies, article et catalogues d'exposition) et de plus de soixante écrits de Charney publiés entre 1962 et 2001 en Amérique du Nord, en Europe et en Asie. 
de ses usages photographiques. Selon Harris, Charney utilise la photographie comme un médium aux fonctions plurielles, intégré au processus conceptuel; elle est «un outil flexible de documentation, d'investigation et d'analyse» (Harris 20). Charney ne mobilise pas la capacité de ce médium à décrire, mais «à révéler comment, dans l'activité de perception, la description est guidée par l'intellect et lui est soumise» (17). Pour autant, et malgré les analyses déjà produites, la généalogie des manières de créer et de penser l'architecture que Charney a développées par la photographie est, jusqu'à récemment, restée méconnue.

Pour les éclairer, nous avons effectué des recherches dans le Fonds Melvin Charney du Centre Canadien d'Architecture (CCA) à Montréal, afin d'examiner, en particulier, les documents produits avant et pendant ses études: c'est à cette époque que Charney amasse les matériaux nécessaires pour forger sa pensée. Une attention particulière a été portée aux scrapbooks de son enfance, aux carnets de notes et de dessins, aux travaux académiques, aux correspondances personnelles et professionnelles et aux matériaux photographiques (diapositives, épreuves, négatifs). Nous avons réexaminé à la lumière de ces documents les écrits publiés par Charney au début des années 1960 (alors qu'il est nouvellement diplômé du Master of Architecture de l'Université Yale), et nous avons réalisé des recherches complémentaires dans les archives de Walker Evans au Metropolitan Museum of Art de New York.

\section{Entre collection et production d'images de la ville}

Charney a lui-même abondamment parlé du rôle essentiel de ses expériences photographiques. Plusieurs de ses notes et commentaires à ce sujet sont rassemblés dans le livre Melvin Charney publié par le Musée d'art contemporain de Montréal sous la direction du conservateur Pierre Landry en 2002, dans le cadre d'une exposition éponyme présentant une importante sélection de ses œuvres photographiques (Landry). Un commentaire de Charney révèle que c'est vers l'âge de dix ans qu'il réalise et développe ses premières prises de vue dans les rues de Montréal, et que cette pratique photographique représente alors pour lui une «manière d'assimiler le monde urbain et industriel en pleine expansion» (23). Il errait alors, précisera-t-il par la suite lors d'une conversation avec la commissaire Yasmeen Siddiqui, comme s'il était en mission d'exploration (Charney 2009, 22).

Charney ne se limite pas à l'observation directe de la ville et de ses matériaux. Il s'intéresse aussi aux collections d'images et à tout ce qu'elles donnent à voir. La consultation de ses archives personnelles permet de constater qu'à l'âge de douze ans, le jeune Melvin amasse des coupures de 
presse, et notamment des articles couvrant des faits à caractère scientifique. Dans ses scrapbooks ${ }^{6}$ se côtoient des articles, des photographies et des illustrations - soigneusement découpés entre 1947 et 1949, parfois pliés, puis collés - concernant l'énergie atomique, le nickel dans les téléviseurs, des découvertes sur les bactéries, les recherches sur le cancer, les oiseaux migrateurs, les nouveaux procédés photographiques, la résistance des matériaux, les parasites, les comètes, une machine faisant fonction de cerveau synthétique capable de penser comme un humain, un laboratoire développant des couleurs de rouges à lèvres, etc. Ses albums contiennent aussi des articles traitant de structures naturelles et primitives, dont des fossiles, des empreintes datant de l'âge de pierre, des micrographies de cellules humaines et de leurs noyaux, soixante-quinze formes de coquillage, etc. S'y ajoutent des images et des textes traitant du thème de la science mise au service de la vie quotidienne: que dire des photographies promotionnelles où est vantée la chaleur radiante d'un plateau en verre capable de faire frire les œufs, griller le pain et réchauffer le café, le tout sans brûler les doigts des ménagères, ou encore d'un nouvel amidon plastique pouvant faire doubler la durée de vie des vêtements féminins? Le contenu des albums fait apparaître les familles d'intérêts que Charney ne cessera d'explorer dans le cadre de sa pratique architecturale et son attachement pour l'origine des formes bâties et naturelles, ainsi que pour les technologies et avancées scientifiques mises au service des gens ordinaires. D'autres éléments témoignent également de sa curiosité intellectuelle, de son esprit critique et de son attention aux productions culturelles populaires. De plus, ces albums sont à l'origine d'une importante méthode de travail de Charney: la collection et la classification de coupures de journaux. Il exploitera par ailleurs ce minutieux procédé dans la réalisation d' «Un Dictionnaire...» dans lequel il rassemble plus de mille quatre cent coupures de journaux avec des photographies où l'on peut voir des bâtiments ou environnements construits comme sujet principal ou en arrière-plan. Dans cette œuvre débutée à l'aube des années 1970 et qui comptera en 2000 deux cent trente-deux planches regroupées en neuf séries, les formes du bâti ordinaire sont pensées par Charney comme les véritables monuments nationaux (Charney 2000) ${ }^{7}$.

6. Newspaper scrapbooks, 1947 et 1949. Fonds Melvin Charney, CCA, nº de dossier: DR2012:0012:088:001; DR2012:0011:012.

L'œuvre «Un Dictionnaire... » a été présentée sous la forme d'une installation, entre autres lieux, à la $7^{e}$ Exposition internationale d'architecture de la Biennale de Venise, en 2000, et à l'Université Harvard, en 1977, sous le titre «Learning from the Wire Services », faisant ainsi référence au livre Learning from Las Vegas de Venturi, Scott Brown et Izenour, paru en 1972. 


\section{La découverte de pratiques visuelles en architecture}

Charney étudie l'architecture à l'Université McGill de 1954 à 1958. Dans un essai de 1956 intitulé «Erich Mendelsohn ${ }^{8}$, il manifeste son intérêt pour les travaux de cet architecte allemand connu pour ses bâtiments expressionnistes et pour ses photographies de la ville américaine, prises en 1924 et publiées en 1926 dans Amerika9 . L'attention qu'il porte à l'œuvre de Mendelsohn n'est probablement pas étrangère aux enseignements de son tuteur Stuart A. Wilson (1912-1991), un jeune architecte montréalais, qui l'ont grandement inspiré. Wilson, qui l'encourage à cultiver son appétit pour l'art, s'occupe par ailleurs de la Sketching School de l'école d'architecture, un atelier dont l'approche est fondée sur l'enregistrement de ce qu'on observe autour de soi (Charney 1999). Wilson accordait ainsi une grande importance aux pratiques visuelles en architecture. Pour sa part, dans son essai sur Mendelsohn, Charney relate que l'architecte allemand avait anticipé l'esthétique industrielle de l'architecture moderne en photographiant les silos et les façades arrière des bâtiments. Ce n'est pas dans les «verticales vertigineuses» des façades des récents gratte-ciels incapables d' «habiller le manque de culture » (Mendelsohn 6) qu'il trouva en Amérique la modernité architecturale qu'il souhaitait observer, mais dans les espaces de servitude, les façades côté ruelle, les ossatures des bâtiments en construction, les installations agricoles, etc. Mendelsohn avait livré, avec son ouvrage Amerika, le portrait photographique d'un itinéraire personnel dans les villes - et les périphéries - de New York, Chicago et Détroit, et ce, en donnant à voir les formes d'une architecture qu'il croyait forte, authentique et résolument fonctionnelle. Comme l'a d'ailleurs souligné Alexandre Rodtchenko en 1928, Mendelsohn avait su photographier "honnêtement» la ville en adoptant la posture de l'homme qui marche dans la rue ${ }^{10}$. Toujours est-il que, lors de son voyage en Amérique, l'architecte allemand se fait très critique envers la façon dont la ville qui «vit du moteur» (Mendelsohn 54) a été pensée. Aussi, il analyse les façades avant comme des représentations grotesques de signes culturels allogènes exploités à tort. Par exemple, des façades d'institutions financières prennent, selon lui, des allures de temples antiques; elles sont à la fois des représentations insouciantes d'un des symboles du pouvoir et les manifestations d'une pauvreté spirituelle. Mendelsohn qualifie aussi d'inhumaine l'échelle des colonnes «décorant» ces institutions tels des silos à argent (92).

Essai écrit par Charney. Fonds Melvin Charney, CCA, ${ }^{\circ}$ de dossier: DR2012:0012:088:005.

Ouvrage traduit en France en 1992, avec une postface de l'historien de l'architecture Jean-Louis Cohen.

10. Propos cités par Jean-Louis Cohen (234), tirés de l'article d'Alexandre Rodtchenko. «Pouti sovremennoï fotografii », Novy Lef 9 (1928): 34. 
Par l'examen d'Amerika, livre où l'auteur commente abondamment ses photographies et documente ses perceptions, Charney découvre l'efficacité des images - de scènes urbaines a priori banales - comme outil de provocation. Dans son essai, il souligne qu'Amerika avait, à sa parution, déstabilisé l'élite architecturale locale:

The complacent downtown architects of New York were shocked out of their seats by this German picture book. It showed only the backsides of the city's most honoured building; it declared that the fire escape, water towers, and blank walls facing the elevated had more architecture in them than the splendid Gothic or Renaissance facade out front. ${ }^{11}$

Après sa découverte d'Amerika, Charney réalise à Montréal, en 1956 et 1957, une grande quantité de clichés qui, pour reprendre ses propres mots plusieurs décennies plus tard, «soulignent les rues et les espaces entre les bâtiments » (Charney 2002, 26). Nombreuses sont les similitudes entre les scènes captées par Charney et celles de Mendelsohn. Les deux hommes orientent leur regard vers la rue et photographient depuis la rue, là où les gens circulent; ils révèlent l'arrière des bâtiments comme lieu véritable de socialisation. Ils photographient les escaliers et les enseignes, objets de la vie quotidienne qui ponctuent le paysage urbain. Toutefois, à la différence de Mendelsohn qui était venu aux ÉtatsUnis en pensant découvrir la ville moderne, Charney ne s'intéresse pas à la modernité architecturale montréalaise. Il la critique et souhaite comprendre la ville différemment de la lecture que propose son institution académique, alors dirigée par John Bland, professeur en histoire de l'architecture. Notons à ce sujet qu'il est hétérodoxe, dans les années 1950 au sein de McGill, de lier approches artistiques et conception architecturale; depuis 1941, un nouveau curriculum moderniste avait été adopté par Bland en remplacement d'un ancien programme basé sur les principes du mouvement Arts and Crafts ${ }^{12}$. Les initiatives artistiques de Charney sont parfois comprises comme des actes provocateurs, comme le démontrent certains commentaires laissés par les évaluateurs de ses travaux ${ }^{13}$. D'après ses témoignages de l'époque, il n'est pas en mesure de discuter franchement avec Bland ni du romantisme en architecture, ni même de l'expressionnisme de Mies Van der Rohe dans les années 1920; en revanche,

11. Essai écrit par Charney. Fonds Melvin Charney, CCA, n ${ }^{\circ}$ de dossier: DR2012:0012:088:005, p. 29.

12. Voir «Highlights of the School's history». URL: https://www.mcgill.ca/architecture/about/featured (page consultée le 5 novembre 2020).

13. Travaux scolaires. Fonds Melvin Charney, CCA, n de dossier: DR2012:0012:088:004 à 014. 
avec Wilson, Charney peut ouvertement partager ses idées personnelles, souvent critiques, quant à la conception architecturale ${ }^{14}$.

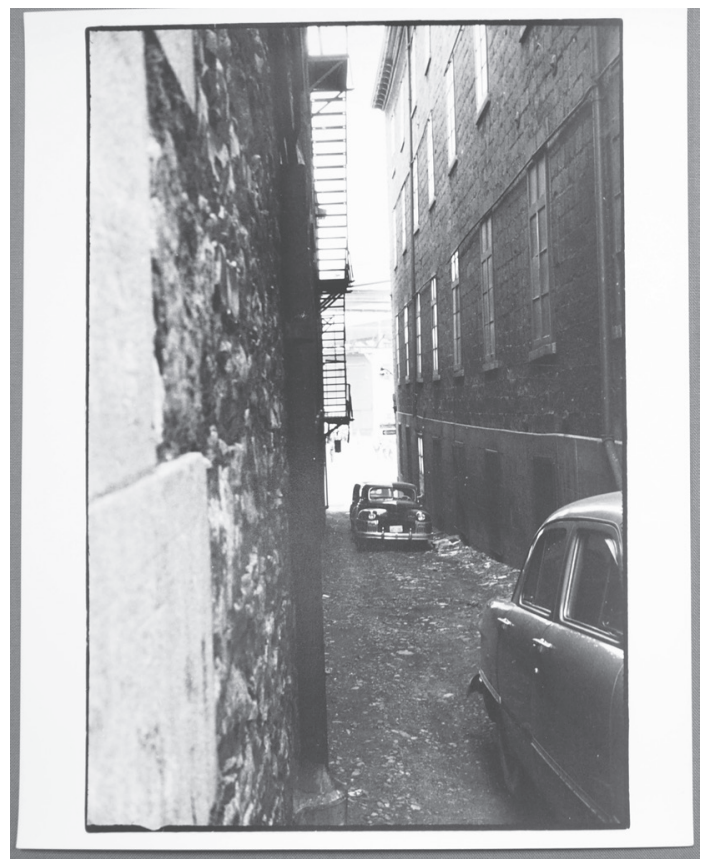

Figure 1. Melvin Charney, Photographie sans titre, ca. 1956-1957.

(C) Succession Melvin Charney / SOCAN (2020). Fonds Melvin Charney, Collection Centre Canadien d'Architecture, Montréal. № de dossier: DR2012:0015:023:004. Photo (C Élène Levasseur/avec l'autorisation du CCA.

Charney cultive de manière de plus en plus marquée le sentiment que les modes de production urbaine développés par ses prédécesseurs, les architectes modernes, sont désuets, et il poursuit ses recherches sur les approches visuelles de l'environnement bâti. Parce que les significations sociales et

14. Idées verbalisées par Charney dans un cahier à reliure noire conservé au CCA où il relate une discussion un 21 octobre. Quelqu'un aurait proposé qu'une forme puisse être conçue avec un certain romantisme. Charney écrit: « There is a complete reaction to this idea. Bland: there is still a potential for fancy in architecture which has its use». Fonds Melvin Charney, CCA, $\mathrm{n}^{\circ}$ de dossier: DR2012:0012:133:005. 
la mémoire collective sont évacuées de cette manière de penser la ville, il se désintéresse du discours architectural dominant qui lui est enseigné. Il se tourne au contraire vers les formes bâties ordinaires et leur capacité à renseigner les dynamiques sociales sous-jacentes à la constitution et aux modifications des formes urbaines.

À la suite de son travail sur Mendelsohn, Charney rédige en 1956 un essai intitulé «Of City and Man $»^{15}$, où il présente les prémisses d'une approche sensible et poétique de la ville basée sur l'observation attentive de l'espace urbain tel qu'il est perçu par l'homme qui s'y déplace. Il insiste notamment sur l'importance d'observer les dimensions sociales des formes urbaines et sur le rôle de l'œil dans l'analyse savante de ces formes. Ce travail est le fruit d'une réelle volonté du jeune architecte de s'informer, de défier les idées préconçues et de trouver des appuis théoriques, en rupture avec les dogmes du mouvement moderne en architecture et en promouvant une pensée architecturale concernée par l'homme et l'espace public de la ville. La bibliographie de ce travail en témoigne. S'y trouvent, entre autres écrits, l'article «Townscape Casebook» de Gordon Cullen, publié en 1949 dans Architectural Review, et plusieurs livres, dont The Things We See d'Alan Jarvis et Vision in Motion de László Moholy-Nagy, tous les deux publiés en 1947, The Culture of the City et The Human Prospect de Lewis Mumford respectivement publiés en 1938 et 1955, ainsi que The Heart of the City: Towards the Humanisation of Urban Life de Jaqueline Tyrwhitt, Josep Lluís Sert et Ernesto N. Rogers, publié en 1952 à l'issue du VIII ${ }^{\mathrm{e}}$ Congrès International d'Architecture Moderne (CIAM 8) qui s'est tenu à Hoddesdon en 1951. Charney illustre aussi son essai avec ses photographies. On constate une familiarité certaine entre son travail photographique et celui de Mendelsohn, en particulier dans le choix des sujets, dans les angles des prises de vue, et dans le style littéraire des légendes. Il écrit en légende d'une de ses photographies : «... from light to dark ... the pressure of enclosed space», tandis que Mendelsohn, traduit de l'allemand par Marianne Brausch, écrivait: «De la lumière à l'ombre, des ténèbres à la lumière - changement continuel, magie de la concurrence» (Mendelsohn 96). Charney écrit encore : « uncontrolled and vulgar and garish public advertising, a scheme for deriving profit from a bare brick wall». De son côté, Mendelsohn soulignait une «confusion grotesque d'architecture construite et plaquée. Des panneaux-réclames hauts comme des maisons sautent dans les espaces résiduels [...]» (Mendelsohn 158).

Par ailleurs, Charney présente d'autres photographies de fragments du paysage urbain, tels des escaliers et un trottoir pavé (Fig. 2). Par ses compositions, il souligne les jeux d'ombres et de lumière et il expérimente des cadrages inhabituels, en recherchant notamment une ligne de fuite, des 
diagonales, etc. Il s'inspire visiblement d'expériences photographiques exposées par Moholy-Nagy dans son ouvrage Vision in Motion (1947).

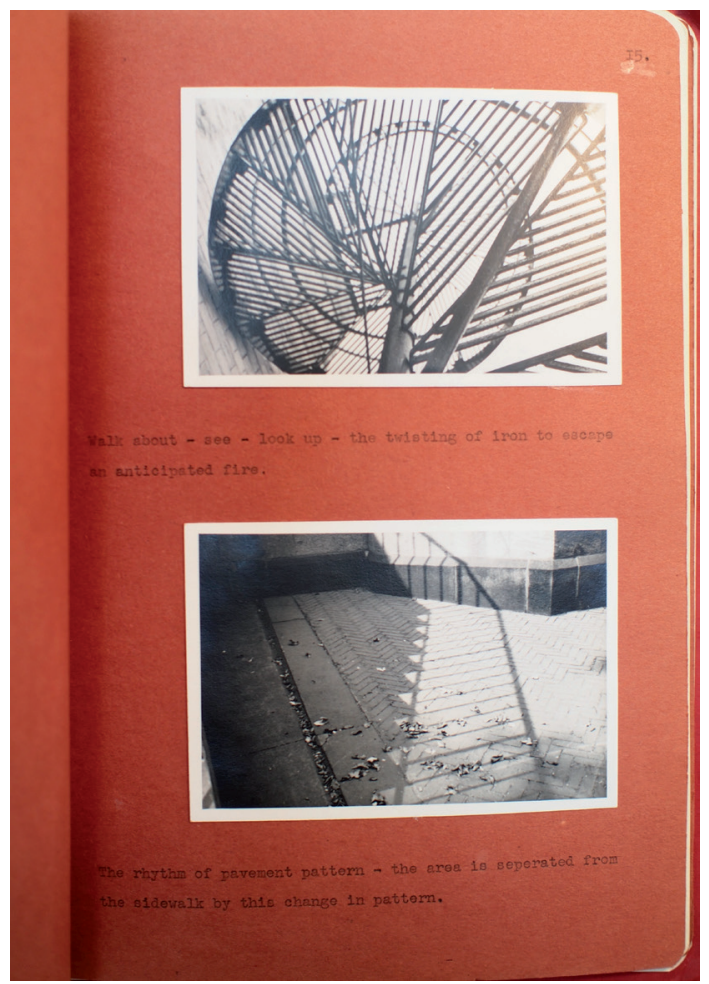

Figure 2. Page 15 de l'essai «Of City and Man», 1956, Melvin Charney. Légendes de Charney: « Walk about see - look up - the twisting of iron to escape an anticipated fire»; «The rhythm of pavement pattern - the area is separated from the sidewalk by this change in pattern».

(C) Succession Melvin Charney / SOCAN (2020). Fonds Melvin Charney, Collection Centre Canadien d'Architecture, Montréal. $N^{\circ}$ de dossier: DR2012:0012:088:009. (C) Élène Levasseur/avec l'autorisation du CCA. 
À cette période, par ses travaux et ses lectures, guidé et encouragé par Wilson ${ }^{16}$, Charney expérimente donc par mimétisme diverses approches photographiques et identifie des références conceptuelles solides sur lesquelles s'appuyer pour développer une pensée originale de l'architecture entendue comme une discipline indissociable des arts visuels.

\section{La découverte des bases honnêtes de l'architecture}

Après son baccalauréat (équivalent de la licence) en architecture à McGill, Charney débute le Master of Architecture à l'Université Yale, à New Haven dans le Connecticut, durant l'été 1958. Il obtient son diplôme en juin $1959^{17}$. Tel qu'en témoignent les brouillons d'une lettre de candidature, rédigée au printemps 1960 dans le cadre d'une demande de bourse d'études à l'Institut royal d'architecture du Canada(IRAC), Charney est alors influencé par les enseignements de Vincent Scully en histoire de l'architecture et par l'enseignement critique du projet architectural dispensé par Louis Kahn. Ce dernier, qui réévalue ouvertement les dogmes de l'architecture moderne et qui est convaincu de l'importance de comprendre les sources historiques des formes architecturales, accompagne Charney dans la construction de sa pensée critique, particulièrement au sujet des aspects culturels et politiques du bâti. Sur les conseils de Kahn - qu'il considère comme son mentor (Martin 442) - et grâce à l'obtention du financement de l'IRAC, Charney part étudier de visu les constructions antiques et moyenâgeuses toujours existantes en Grèce, en Turquie, en Italie et en France. Dans sa lettre de candidature, il soutient que les observations faites sur le terrain peuvent contribuer à sa future pratique de l'architecture. Selon lui, l'étude de l'histoire sert à découvrir et résoudre des problèmes qui ont toujours existé - par exemple, en identifiant une gamme de réponses formelles à travers l'expression d'éléments architecturaux basiques, dont les colonnes : «Observe at Paestum how a series of stone elements become a column. How does concrete become a column? What form does a concrete column assume? ».

Dans une lettre datée du 2 août 1960 adressée à ses parents ${ }^{18}$, Charney mentionne le fait qu'il étudie des photographies d'architectures anciennes pour préparer son voyage. Il réalise plusieurs dessins

16. Charney a mentionné lors d'un entretien à McGill en 1999 que c'est dans cette université qu'il a forgé ses idées, en grande partie grâce aux enseignements précieux de Wilson (Charney 1999).

17. Information mentionnée par Charney dans un brouillon de sa lettre de candidature pour la bourse du Royal Architectural Institute of Canada, au printemps 1960. Fonds Melvin Charney, CCA, ${ }^{\circ}$ de dossier: DR2012:0012:088:022. Les informations présentées dans ce paragraphe proviennent de ce brouillon.

18. Lettre de Charney à ses parents le 2 août 1960. Fonds Melvin Charney, CCA, $n^{\circ}$ de dossier: DR2012:0012:088:018. 
de détails architecturaux dans des carnets ${ }^{19}$, note les matériaux utilisés et la fonction de différentes composantes architecturales qu'il observe dans ces images photographiques, dont certaines ont été publiées dans des brochures touristiques. La collecte de documents ayant trait à l'ordinaire est restée chez Charney une manière d'accéder à un savoir, et ce depuis l'enfance, alors qu'il confectionnait des scrapbooks. Il utilise notamment une photographie trouvée pour faire l'esquisse d'une maison typique des villages à l'est de Konya, en Turquie. Ses murs porteurs ont été construits en adobe et sont parfois recouverts d'un stuc blanc, tandis que des troncs d'arbres servent de linteaux. Quelques mois plus tard, lors d'un séjour sur place, en 1961, il repère sur la route entre Konya et Aksaray cette maison qu'il avait dessinée. Il la photographie frontalement, de manière à mettre en évidence les éléments architecturaux préalablement identifiés.

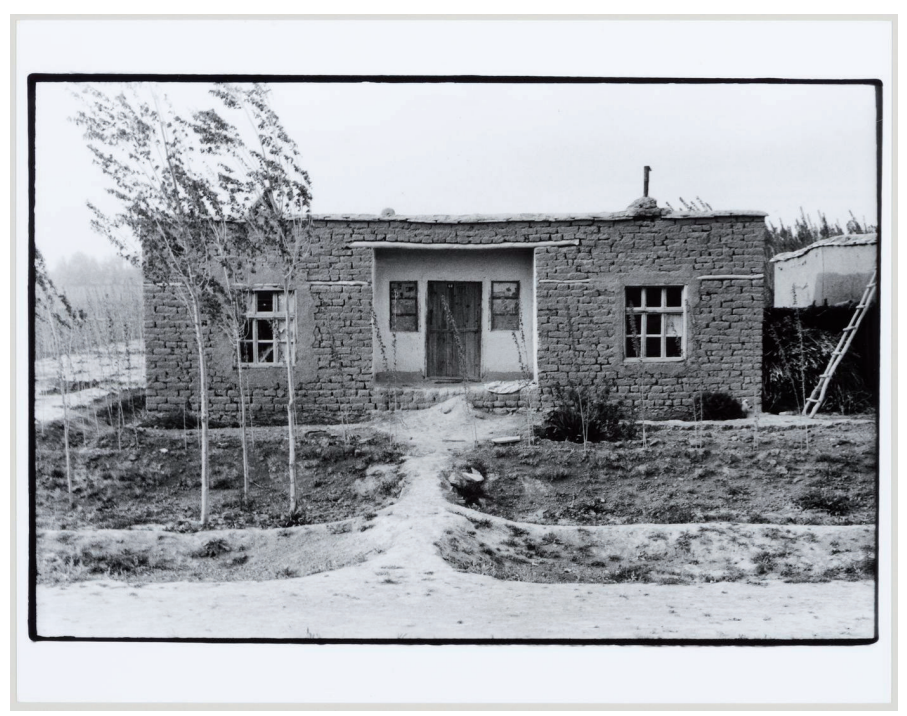

Figure 3. Melvin Charney, House, road between Konya and Aksaray, Turkey,1961.

(C) Succession Melvin Charney / SOCAN (2020). Fonds Melvin Charney, Collection Centre Canadien d'Architecture, Montréal. Nº de référence: PH2002:0021, dossier DR2012:0012:081:005. 


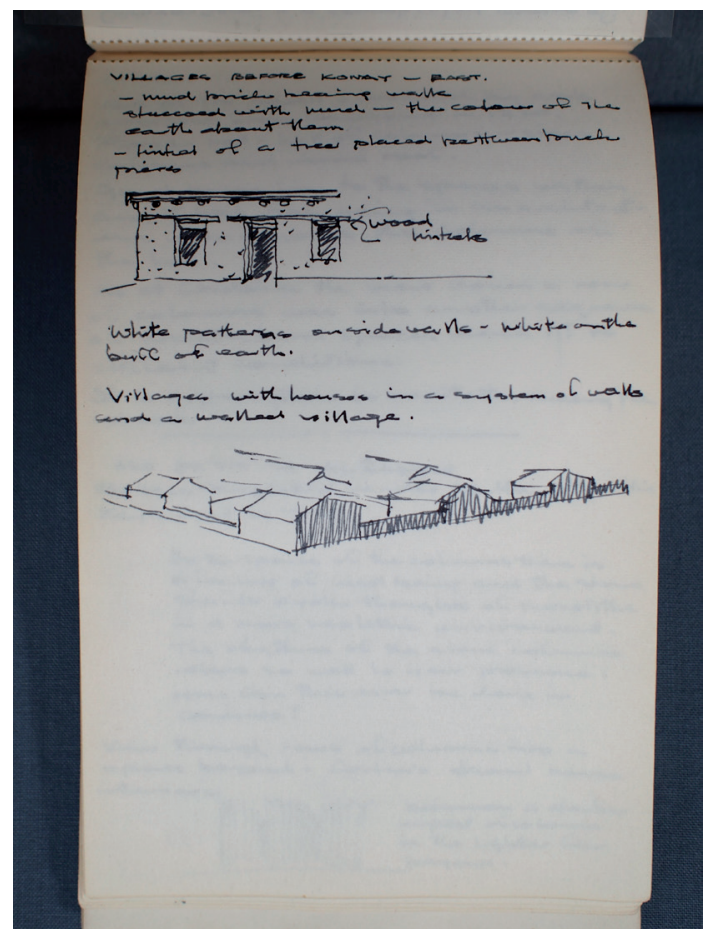

Figure 4. Page d'un carnet de notes de Charney, 1960.

(C) Succession Melvin Charney / SOCAN (2020). Fonds Melvin Charney, Collection Centre Canadien d'Architecture, Montréal. No de dossier: DR2012:0011:010. Photo C Élène Levasseur/avec l'autorisation du CCA.

Une grande quantité de photographies, diapositives et planches-contacts conservées au CCA ${ }^{20}$ donne à voir des architectures religieuses et, surtout, des architectures vernaculaires. Charney, dans un commentaire publié dans la monographie que lui a consacrée le Musée d'art contemporain de Montréal, dit avoir eu tendance à éviter les monuments célèbres à l'exception de quelques temples de la Grèce antique, et avoir été davantage attiré «par des lieux qui demeurent hors du temps et de

20. Une trentaine de photographies, toutes prises en 1961, est également reproduite dans le livre consacré à ses expériences photographiques qui est publié par le Musée d'art contemporain de Montréal (Landry). 
l'histoire» (Charney 2002, 37). À ce sujet, dès son retour, Charney publie plusieurs articles portant sur ses observations et apprentissages réalisés au cours de son voyage dans le bassin méditerranéen, dont «A Journal of Istanbul: Notes on Islamic Architecture» paru en juin 1962 dans le Journal of the Royal Architectural Institute of Canada (l'IRAC). Charney y écrit en introduction: «Buildings and cities, ancient architectures, pleasing handcrafted work, sheds and cathedrals are all part of another world - a huge anonymous collection which one can still visit to find origins and motives of architecture, and delight in its forms » (Charney 1962, 60). Il relate avoir observé des formes architecturales, leurs articulations, leurs répétitions et leurs interrelations. Il considère que l'arrangement spatial d'une construction génère une image mentale dans l'esprit de celui qui la visite; il parle en termes «de post-image, montage d'impressions visuelles» (Charney 1962, 64). Dès lors, Charney constate que le vocabulaire architectural rend des types de bâtiments, dont les lieux de culte, reconnaissables par les membres de communautés données. Ainsi, des éléments architecturaux devenus familiers sont réutilisés depuis des siècles. Les pratiques de répétition d'éléments architecturaux reconnaissables de tous ont été enseignées à Yale, entre autres par James Stirling ${ }^{21}$, architecte britannique que Charney a identifié comme une des personnes l'ayant influencé lors de son master. Stirling développait alors l'idée d'un vernaculaire régional, et la photographie du bâti était pour lui un moyen de découvrir la grammaire qui dicte la conception de détails architecturaux signifiants. D'une certaine manière, les formes et structures vernaculaires étaient pour lui des instruments méthodologiques qui lui permettaient, explique-t-il en 1987, de mieux comprendre ce qui importe aux gens qui les habitent et ce qui convient au site où elles sont érigées (Maxwell 229). En 1960, Stirling a d'ailleurs mis en évidence, dans son article «The Functional Tradition and Expression» publié dans le journal Perspecta: The Yale Architectural Journal, que la recherche sur les formes vernaculaires avait eu des conséquences sur l'architecture moderne. Notamment, il y analyse, en images, la chapelle de Ronchamp, icône du style tardif de Le Corbusier. Il fait correspondre une de ses propres photographies de Ronchamp et une photographie anonyme d'une habitation médiévale, en soulignant l'évidente parenté entre les formes de ces architectures (Stirling, 1960, 88-89). Il souligne ainsi, par l'association d'images, la ressemblance entre des détails conçus par Le Corbusier et une construction populaire observée dans un village. Depuis la Seconde Guerre mondiale, Le Corbusier délaissait progressivement l'esthétique de l'ère des machines et s'intéressait davantage à la réalité quotidienne de l'homme, aux formes de l'imaginaire et au volumes significatifs et symboliques (Le Corbusier).

21. L'examen de notes utilisées par Stirling lors de ses enseignements à Yale, où certains passages sont soulignés et d'autres écrits en lettres majuscules ou en couleur, permet d'identifier les notions sur lesquelles il insistait. Fonds James Stirling/ Michael Wilford, CCA, $n^{\circ}$ de référence: AP140.S2.SS4.D7.P5.2; AP140.S2.SS4.D7.P5.7. 
Toujours dans la continuité de son voyage dans le bassin méditerranéen, Charney publie deux articles dans Landscape, le périodique fondé en 1951 par John Brinckerhoff Jackson (1909-1996), pionnier de l'étude du paysage - concept qu'il définit dans son versant politique comme produit concret du pouvoir établi et dans son versant vernaculaire comme espace aménagé par et pour les hommes ordinaires. L'article «Troglai: Rock-Cut Architecture» paraît en 1963 et le second, «The Trulli of Southern Italy», en 1965 (Charney 1963 et 1965b). Si dans son premier texte Charney demeure très descriptif, dans celui de 1965, il critique ouvertement les principes modernistes alors en vigueur. Ce dernier texte, par ailleurs traduit et publié en 1965 dans la revue Vie des arts sous le titre «Architecture sans architectes: les trulli de l'Apulie» (Charney 1965a), paraît alors qu'est montrée au Musée d'art moderne de New York (MoMA) de novembre 1964 à février 1965 l'exposition conçue par l'architecte Bernard Rudofsky, Architecture Without Architects : A Short Introduction to Non-Pedigreed Architecture. L'exposition présente quelque deux cents images d'habitations traditionnelles de plus de soixante pays. Comme il s'était intéressé à Amerika de Mendelsohn, Charney s'enthousiasme pour cette entrée des images de l'architecture de l'ordinaire dans un lieu prestigieux. Il ne manque pas de souligner dans son article de 1965 que les images photographiques présentées ont été tirées de livres illustrés et d'albums souvenirs de photographes amateurs. Il met également en évidence le potentiel des faits architecturaux présentés comme objets de savoir. Selon lui, ce qui est le plus intéressant dans l'observation de formes architecturales vernaculaires, «ce sont les valeurs humaines qui ont persisté ici en dépit des changements et qui gardent toute leur force en un temps où l'humanité se détache de plus en plus de son milieu naturel» (Charney 1965a, 57). Dans une version préliminaire de ses articles de $1965^{22}$, il mentionne l'opportunité, en particulier pour les architectes, de découvrir des systèmes environnementaux forts et clairs permettant des variations fonctionnelles, et ce, au sein même d'une méthode de construction standard. C'est un aspect que Charney avait abordé en 1962 dans «A Journal of Istanbul: Notes on Islamic Architecture», mais grâce à «Architecture Without Architects», il a maintenant l'occasion de valider ses analyses et d'étayer ses propos. Non seulement Rudofsky institutionnalise l'architecture primitive, mais il élève également la photographie du bâti ordinaire au statut de pratique savante permettant d'étudier et de découvrir les bases honnêtes de l'architecture.

22. Ses articles publiés en 1965 avaient été abrégés. L'article, dans sa forme originale, a été mis au jour en 2013 dans l'anthologie des écrits de Charney éditée par Louis Martin (115). 


\section{La photographie de l'ordinaire comme objet du savoir}

Dans le sillage de l'exposition conçue par Rudofsky, Charney continue à se passionner pour la photographie comme outil de la production de connaissance architecturale. Il poursuit, comme il le faisait à McGill, ses recherches sur les modes opératoires photographiques qui sont institutionnellement reconnus et sur lesquels il peut s'appuyer. Dans sa quête, il sollicite les conseils de Walker Evans - fait qui était resté inconnu par ses proches collaborateurs lorsque nous les avons rencontrés entre 2013 et 2018 à l'Université de Montréal. Les recherches dans le fonds de Charney, pourtant très bien garni, ne permettent pas d'en apprendre davantage au sujet de leur relation. Par contre, lors d'allocutions prononcées en 1996 et $1999^{23}$, Charney mentionne le nom d'Evans parmi ceux qu'il a côtoyés et qui l'ont influencé à Yale. Nous avons entrepris des recherches dans les archives d'Evans au Metropolitan Museum of Art pour éclaircir ce point. Si tous ses agendas et cahiers de notes de 1959 (année de l'arrivée de Charney à Yale) à 1964 (année où Evans a commencé à enseigner à Yale) ont été consultés en vain, l'examen des cahiers issus des enseignements d'Evans a toutefois mené à la découverte de documents de travail portant traces de leurs échanges entre l'automne 1965 et l'hiver 1966. Quelques notes d'Evans dans un petit carnet bleu et une feuille de papier pliée qui y est insérée ${ }^{24}$ attestent de la présence de Charney au séminaire d'Evans à l'automne 1965. Comme en témoignent les notes d'Evans, il a été question, dans un projet photographique proposé par Charney, d' " anonymous graphies» et de «hand signs Montreal» ${ }^{25}$. Charney pourrait avoir présenté des photographies semblables à celle qu'il a prise en 1964 d'une façade de la rue Marie-Anne à Montréal, présentant un bâtiment placardé d'enseignes publicitaires peintes à la main. Ayant une bonne culture artistique, il est probable qu'il connaissait déjà la passion d'Evans pour les panneaux et affiches dans lesquelles un savoir-faire manuel et populaire est consigné.

23. Charney nomme Evans dans un discours prononcé en février 1996, CCA / Oxford-Cambridge Lecture (transcription repérée dans le Fonds Melvin Charney, CCA, $n^{\circ}$ de dossier: DR2012:0012:103:023), et dans un entretien avec Jim Donaldson trois ans plus tard (Charney 1999).

24. Archives de Walker Evans, Metropolitan Museum of Art, $\mathrm{n}^{\circ}$ de référence: 1994.250.144.

25. Ces appellations apparaissent respectivement le 10 et le 22 janvier 1966 dans le carnet bleu, Metropolitan Museum of Art, $\mathrm{n}^{\circ}$ de référence: 1994.250 .144 . 


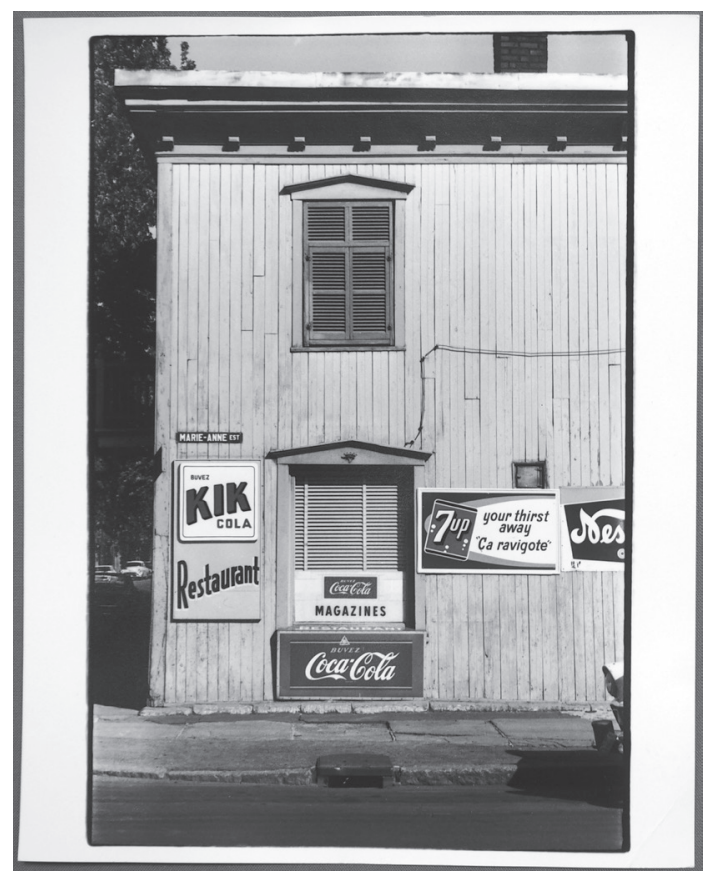

Figure 5. Melvin Charney, Sans titre, 1964.

(C) Succession Melvin Charney / SOCAN (2020). Fonds Melvin Charney, Collection Centre Canadien d'Architecture, Montréal. $N^{\circ}$ de dossier: DR2012:0015:023:004. Photo (C) Élène Levasseur/avec l'autorisation du CCA.

L'article «Experimental Strategies: Note for Environmental Design» publié en 1969 dans Perspecta: The Yale Architectural Journal, atteste des analyses que développe Charney, à l'aube des années 1970, afin de faire participer la photographie à la construction et à la diffusion de discours architecturaux. Charney y mentionne l'étude récemment entreprise à Las Vegas par Robert Venturi et Denise Scott Brown (il ne signale pas la participation de Steven Izenour), dans le cadre de laquelle la photographie était utilisée comme un instrument de recherche sur la morphologie de la rue commerciale. Charney reprend d'ailleurs dans son texte la première phrase de l'article «A Significance for A\&P Parking Lots or Learning from Las Vegas» que Venturi et Scott Brown publient en 1968 et qui sera précurseur de Learning from Las Vegas, paru en 1972: «Learning from the existing landscape is a way 
of being revolutionary for an architect» (Venturi et Scott Brown 1968, 37). Les deux auteurs précisent toutefois dans leur article qu'il ne s'agit pas d'être révolutionnaire au sens de Le Corbusier proposant de raser Paris et de tout recommencer, mais d'être révolutionnaire d'une manière plus tolérante par rapport à l'existant. Cette manière de faire s'est traduite par l'analyse de la rue comme un document de la vie quotidienne informant sur l'esthétique de la société de consommation. Charney trouve cette approche du Strip des plus inventives (Charney 1969, 25). Outre la posture de Venturi et Scott Brown, il présente dans son article la photographie «6565 Fountain Ave.» que l'artiste conceptuel Edward Ruscha a publiée en 1965 dans Some Los Angeles Apartments. S'appuyant sur cette photographie, il insiste sur l'absurde maniérisme de 1' "Architecture», avec un grand A, des façades des rues commerciales qu'il analyse comme l'emballage d'un fait urbain consommable (27). Plus avant, l'article «Experimental Strategies» témoigne de l'intérêt de Charney pour le travail de Ruscha. Cet intérêt est tel que l'architecte montréalais se procure au moins quatre de ses livres d'artiste: Twenty-Six Gasoline Stations, 1967 (la première édition datait de 1963); Some Los Angeles Apartments, 1965 ; Thirty-Four Parking Lots in Los Angeles, 1967 ; et Nine Swimming Pools and a Broken Glass, $1968^{26}$. Dans ces quatre ouvrages, Ruscha présente trivialement et sans artifice la banalité du cadre bâti étatsunien; il laisse la scène parler d'elle-même. Ses photographies sont des documents d'un avoir-été présentant des constructions faites de matériaux standardisés et qui portent les signes d'une société contrôlée par les technologies. Sous d'autres rapports, tout comme Charney d'ailleurs, Ruscha a été profondément bouleversé par la photographie d'Evans à la fin des années 1950 (Ruscha 205).

Toujours en lien avec l'intérêt de Charney pour l'œuvre photographique de Ruscha, soulignons que dans le cadre de l'exposition «Montréal, plus ou moins?» qui s'est tenue au Musée des Beaux-arts de Montréal en 1972, Charney présente l'œuvre «The Main... », un panorama composé de vingt-sept photographies coupées et ajustées du boulevard Saint-Laurent. Le montage photographique de Charney reprend à la lettre la grammaire de «Every Building on the Sunset Strip», que Ruscha produit en 1966 et dont Charney présente un segment dans son article «Experimental Strategies» trois ans plus tard. Rappelons aussi que Venturi, Scott Brown et Izenour, ainsi que leurs étudiants de l'atelier Learning from Las Vegas organisé à l'automne 1968 dans le cadre de l'École d'art et d'architecture de Yale, sont eux aussi influencés par les dispositifs photographiques de Ruscha. L'un des étudiants, Douglas Southworth, qui a personnellement rencontré Ruscha dans son studio de Los Angeles (Salomon et Kroeter), produit un panorama à la manière de «Every Building on the Sunset Strip». Prises depuis le centre de la rue, les images photographiques révèlent certains aspects de la ville qui n'avaient auparavant presque jamais suscité de débats dans le milieu architectural. Elles montrent notamment les relations 
souvent chaotiques entre les multiples éléments qui se juxtaposent dans le paysage urbain. Enfin, pour Charney, comme pour Ruscha, Venturi et Scott Brown, la rue commerciale et son architecture sont des expressions de la société de consommation et la photographie contribue à leur étude.

Il faut mentionner que les thèmes abordés par Charney s'inscrivent dans un plus vaste courant. Dans les années 1960 et 1970, des photographes créent de nouveaux rapports artistiques au réel: ils mettent au jour des lotissements populaires, des quartiers en ruine ou réhabilités, des bâtiments d'usine, des installations nucléaires, des cultures extensives, de grandes artères urbaines, des motels, des parkings,

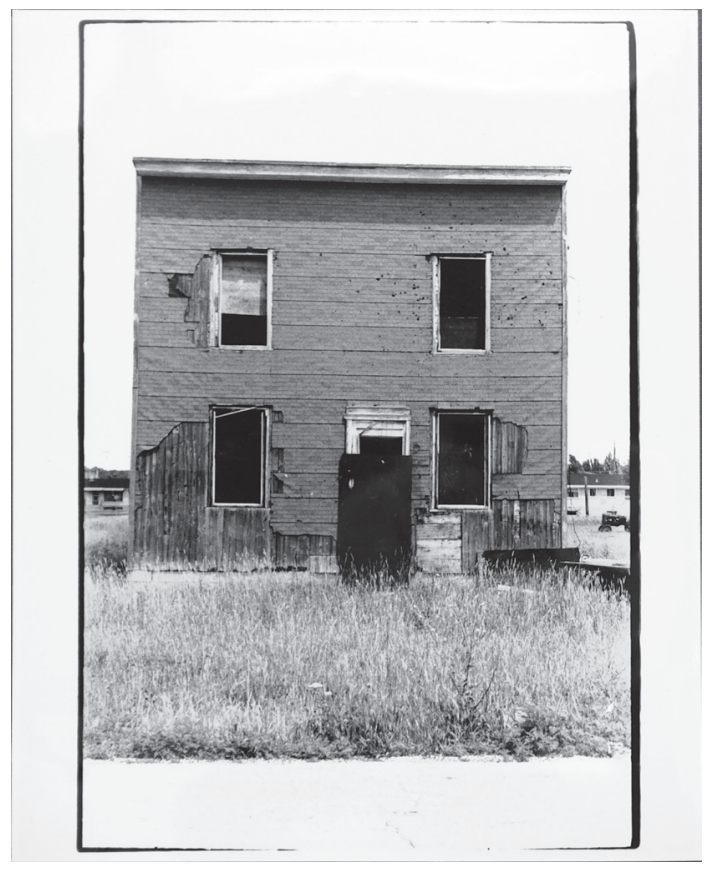

Figure 6. Melvin Charney, Maison, Rivière-des-

Prairies, Québec, 1975.

(C) Succession Melvin Charney / SOCAN (2020).

Fonds Melvin Charney, Collection Centre Canadien d'Architecture, Montréal. $\mathrm{N}^{\circ}$ de dossier: DR2012:0015:023:004. Photo (C) Élène Levasseur/avec l'autorisation du CCA. $\operatorname{etc}^{27}$. En revanche, selon Charney, l'architecture évolue toujours dans une atmosphère dominée par une idée de la modernité d'origine européenne et où l'histoire de l'architecture populaire n'est guère valorisée par les institutions académiques et politiques. Lors d'une allocution aux Conférences J.A. de Sèves qui se sont tenues à Montréal en 1971, Charney dénonce l'institutionnalisation des références et plus largement des signes architecturaux qui rendent l'architecture inaccessible aux gens du peuple. Il est temps, selon lui, «que l'on essaie de faire le point sur l'architecture québécoise» (Charney 1971, 11).

Ainsi, Charney se dote publiquement d'une mission: apprendre du paysage construit et des relations subjectives entre l'homme et son environnement tels qu'ils sont rendus perceptibles par une lecture attentive de ce qui est déjà là. Dès lors, Charney produit lui-même un imposant corpus de photographies d'humbles constructions qu'il a identifiées dans le vaste territoire canadien s'étendant au-delà des zones densément peuplées. Il se passionne pour les traces des transformations

\footnotetext{
27. Voir notamment l'exposition «New Topographics» à la George Eastman House à Rochester dans l'État de New York en 1975-1976.
} 
physiques du bâti, dont les vestiges d'anciennes ouvertures, et les étudie pour mieux comprendre, entre autres sujets, les changements d'usages des bâtiments. De son travail résulte une imagerie savamment composée - l'architecte semble avoir intégré des manières de faire d'Evans et de Ruscha. Manifestement, il s'intéresse en priorité à l'histoire des marges, de l'ordinaire, du populaire, du quotidien. Quelques décennies plus tard, il suggèrera d'ailleurs dans un commentaire sur ses propres photographies «que les rudes conditions de vie en marge exposent les "véritables" forces et mutations qui résident dans des rapports bien camouflés» (Charney 2002, 54).

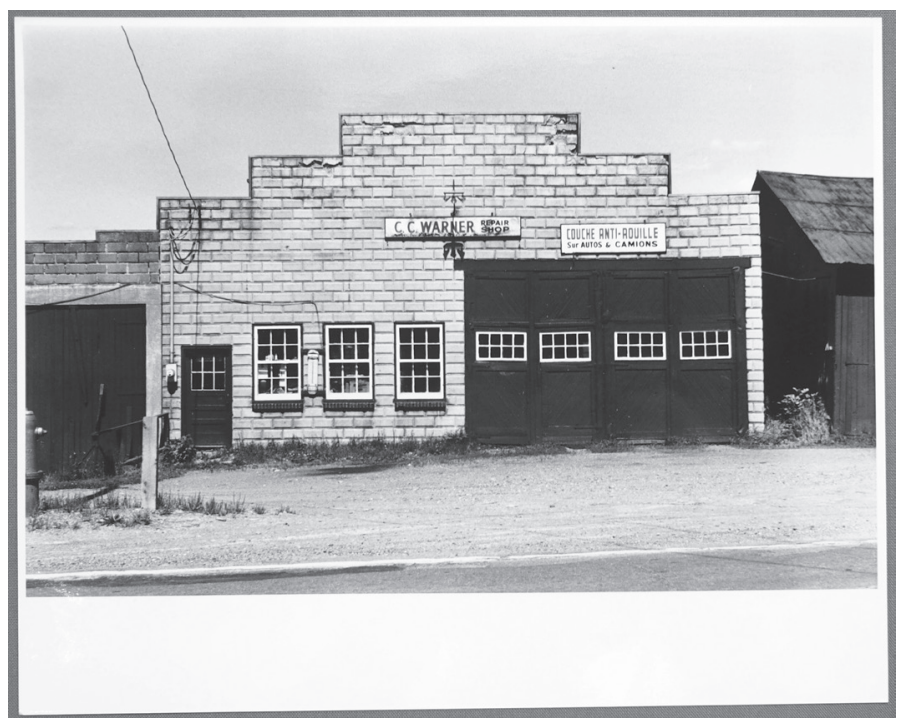

Figure 7. Melvin Charney, Garage, Lennoxville, Québec, 1977.

(C) Succession Melvin Charney / SOCAN (2020). Fonds Melvin Charney, Collection Centre Canadien d'Architecture, Montréal. $\mathrm{N}^{\circ}$ de dossier: DR2012:0015:023:004. Photo (C) Élène Levasseur/avec l'autorisation du CCA. 


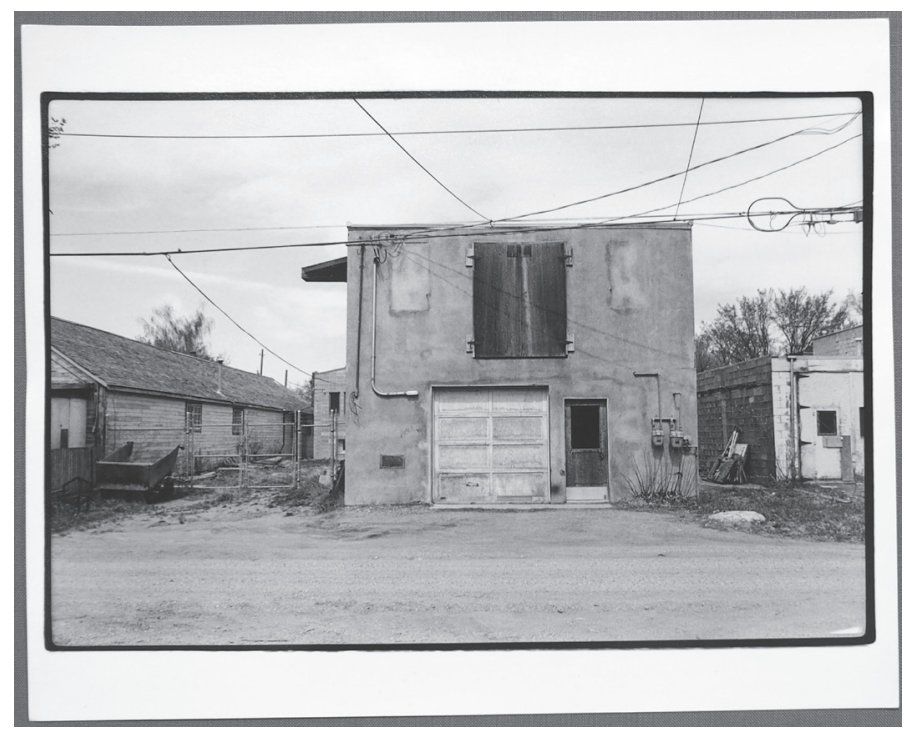

Figure 8. Melvin Charney, Garage, Saskatoon, Canada, 1988.

(C) Succession Melvin Charney / SOCAN (2020). Fonds Melvin Charney, Collection Centre Canadien d'Architecture, Montréal. $N^{\circ}$ de dossier: DR2012:0015:023:004. Photo C Élène Levasseur/avec l'autorisation du CCA.

En parallèle, Charney continue d'amasser des cartes postales et d'autres images d'architectures anonymes et populaires datant de la première période d'industrialisation du Québec (années 1870 à 1920), tirées de livres de photographies et de brochures touristiques et gouvernementales, pour ne citer que ces seules sources. Comme Clément Chéroux le suggère à propos d'Evans (Chéroux 9), le vernaculaire est pour Charney à la fois un sujet dans l'espace bâti et une méthode visuelle fonctionnant par appropriation de formats non artistiques. À la manière d'Evans collectionnant des panneaux, l'architecte collecte également quelques fragments de matière provenant de la démolition de bâtiments (Charney 1977). Charney rassemble plusieurs éléments sur le mur de son atelier, dont des photographies de modestes maisons, de temples grecs et d'églises, ainsi que le livre American Photographs d'Evans, ouvert aux pages 134-135; on y voit la photographie «Country Store and Gas Station, Alabama, 1936», qui représente une station-service munie d'un fronton ornemental. Charney a en quelque sorte créé un dictionnaire visuel, voire une matériauthèque vernaculaire de ce qui informe l'architecture québécoise et canadienne - une figuration nouvelle de la maison, des ruines, des signes et des formes de la hutte 
primitive (Charney 1982). Qui plus est, ce mur peut être entendu comme une cartographie de ses relations visuelles et historiques à l'architecture, et de la manière dont il les a progressivement définies.
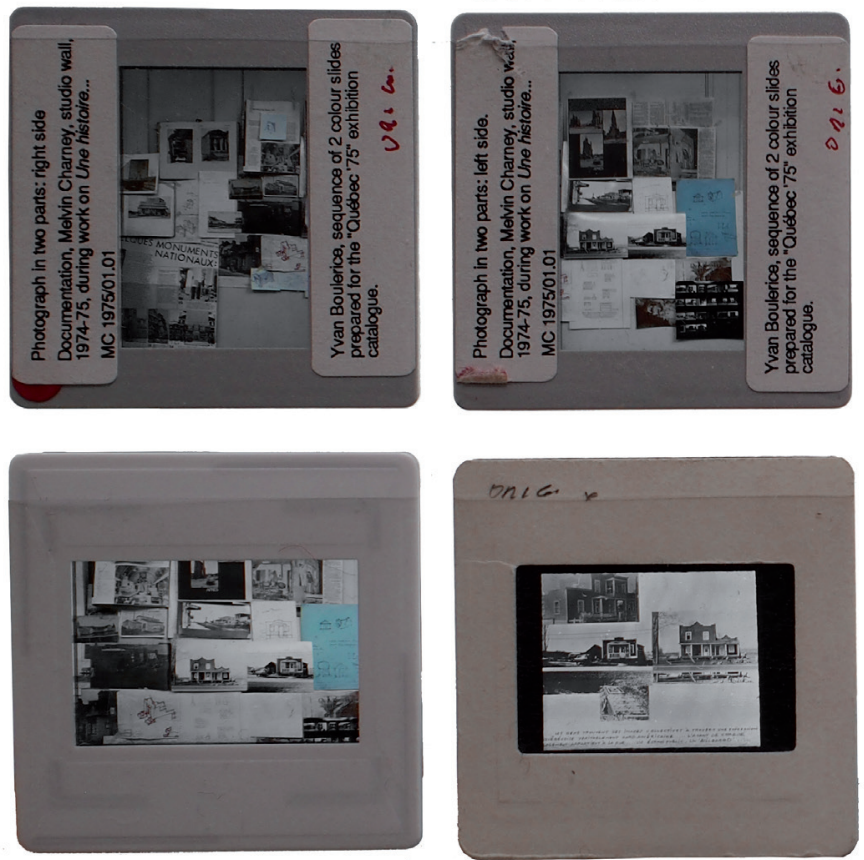

Figure 9. Yvan Boulerice, Documentation, Melvin Charney, studio wall, 1974-75, during work on Une histoire..., 1975.

(C) Fonds Melvin Charney, Collection Centre Canadien d'Architecture, Montréal. $\mathrm{N}^{\circ}$ de dossier : DR2012:0012:083:001. Photo (C Élène Levasseur / avec l'autorisation d'Yvan Boulerice et du CCA.

La démarche visuelle et historique que Charney a développée ne peut pas être dissociée de son désormais célèbre article publié pour la première fois en 1980 , « Montrealness of Montreal» ${ }^{28}$

28. Article initialement publié en 1980 dans The Architectural Review 999 (mai 1980) : 299-302. La version consultée est une traduction de Charney parue en 1992 sous le titre de «Montréal: Formes et figures en architecture urbaine» (Charney 1992). 
- titre qui pourrait être traduit par «la Montréalité de Montréal». Dans cet article, Charney s’intéresse à l'image et à la spécificité des formes urbaines montréalaises. Il les décompose et commente leurs systèmes de références sociales et historiques, pour comprendre leur genèse. Charney décrit dans son texte les différentes couches de mémoire qui constituent la ville, elle-même document du métissage des cultures française et britannique et «des courants architecturaux communs à tous les empires européens» (Charney 1992, 18). Puis, avec les «Colonnes allégoriques» qu'il conçoit et réalise de 1987 à 1990 pour le jardin du CCA, Charney figure les composantes essentielles du site, enclavé entre le boulevard René-Lévesque et l'autoroute Ville-Marie, qu'il compare à celui des Buttes-Chaumont, puisqu'il a été anciennement occupé par une carrière, un abattoir et des voies ferrées (Charney 1998, 121). Il puise en quelque sorte au sein du site des images enfouies dans ses différentes couches de mémoire. Entre autres éléments, sont figurés dans ces colonnes les églises à deux clochers et les structures des aciéries des quartiers adjacents; les maisons québécoises et les logements ouvriers qu'il a par ailleurs abondamment photographiés; les silos à grains symbolisant à la fois le port de Montréal et le mouvement moderne (et élitiste) en architecture; un «temple-silo» qui rappelle par ailleurs les commentaires cinglants de Mendelsohn sur les banques américaines aux allures de temples grecs et sur leurs colonnes qu'il dépeint comme des silos à argent (Mendelsohn 92). Charney articule ainsi les lexiques de deux discours architecturaux distincts: l'un est dérivé de la théorie et des formes de l'architecture moderne, tandis que l'autre provient de sa lecture sensible de la ville comme produit et canevas de la vie des gens ordinaires.

\section{La photographie du vernaculaire comme levier de la pensée critique}

L'examen des travaux académiques de Charney et de certaines de ses œuvres démontre que la photographie comme pratique et document ayant trait à l'architecture vernaculaire a été pour lui un outil d'articulation et d'affirmation d'une pensée architecturale critique forgée en rupture avec celle des architectes modernes. L'imagerie architecturale qu'il a produite est, à plusieurs égards, comparable à celle du bâti vernaculaire étatsunien développée par Evans dans les années 1930, et à celle de Ruscha, qui était son contemporain. Elle représente une architecture du quotidien qui, bien que banale, apparait fondamentale, voire monumentale, pour les gens ordinaires. Parallèlement, grâce à ses analyses photographiques et documentaires, Charney a su identifier les traits caractéristiques, et parfois même génériques, de constructions relevant de l'ordre du banal et de l'ordinaire. Puis, en les mettant en lumière et en les faisant entrer dans les institutions, il a élevé les manières de faire vernaculaire au rang d'objets de connaissance. En cela, Charney s'est intéressé, au tournant des années 1970, «à la notion de la figuration comme une dimension du savoir et de la mémoire collective qui imprègne 
l'acte de bâtir» (Charney et Lambert 27). Il a cherché à définir les caractéristiques constitutives réelles des monuments, à mieux cerner l'identité de l'architecture québécoise et à mieux comprendre ce qui structure l'environnement bâti - physiquement, humainement, culturellement, institutionnellement et politiquement parlant. En ce sens, le jardin du CCA pourrait être considéré comme la consécration de sa démarche: les colonnes et les autres objets qu'il a mis en relation sur le site, dont un verger et des répliques d'éléments architecturaux de la Maison Shaughnessy (une résidence de style Second Empire intégrée au CCA) constituent des figurations nouvelles d'une mémoire collective tournée vers les formes bâties propres au lieu. Le jardin est allégorique: il révèle et institutionnalise, comme le mentionne Charney, «les thèmes primaires d'une iconographie contemporaine» (Charney 2002, 86).

Finalement, au-delà de l'éclairage nouveau apporté sur la généalogie de l'œuvre de Charney et du potentiel de la photographie du vernaculaire comme mode d'accès à un savoir architectural, il importe de retenir qu'une expérience photographique menée en toute liberté intellectuelle est le levier d'une pensée originale et critique. Pour penser et inventer ce qui reste à construire, il faut commencer par apprendre à regarder et comprendre honnêtement ce qui est déjà là.

\section{OUVRaGes CITÉS}

CHARNEY, Melvin. «A Journal of Istanbul: Notes on Islamic Architecture». Journal of the Royal Architecture Institute of Canada 39.6 (1962): 60-64.

CHARNEY, Melvin. «Troglai: Rock-Cut Architecture». Landscape 12.3 (printemps 1963): 6-12.

CHARNEY, Melvin. «Troglai : Architecture troglodyte en Cappadoce». Vie des arts 34 (1964) : 46-52.

CHARNEY, Melvin. «Architecture sans architectes : les trulli de l'Apulie». Vie des arts 38 (1965a): 54-58.

CHARNEY, Melvin. «The Trulli of Southern Italy». Landscape 15.1 (1965b): 32-33.

CHARNEY, Melvin. «Experimental Strategies: Notes for Environmental Design». Perspecta: The Yale Architectural Journal 12 (1969): 21-29.

CHARNEY, Melvin. «Pour une définition de l'architecture au Québec». Architecture et urbanisme au Québec. Dir. Melvin Charney et Marcel Bélanger. Montréal: Les Presses de l'Université de Montréal, 1971. 9-42.

CHARNEY, Melvin. «Other Monuments : Four Works, 1970-1976». Vanguard 6.2 (mars 1977) : 3-8.

CHARNEY, Melvin. Melvin Charney: Euvres, 1970-1979. Montréal : Musée d'art contemporain de Montréal, 1979.

CHARNEY, Melvin. «Montrealness of Montreal». The Architectural Review 999 (mai 1980) : 299-302. 
CHARNEY, Melvin. «À qui de droit: au sujet de l'architecture contemporaine au Québec». ARQ ArchitectureQuébec 5 (janvier-février 1982): 12-23.

CHARNEY, Melvin. Paraboles et autres allégories : l'œuvre de Melvin Charney, 1975-1990. Montréal: Centre canadien d'architecture, 1991.

CHARNEY, Melvin. «Montréal: Formes et figures en architecture urbaine». 1980. Ville Métaphore Projet Architecture urbaine à Montréal: 1980-1990. Dir. Irena Latek. Montréal: Éditions du Méridien, 1992. 17-30.

CHARNEY, Melvin. «La cité incontournable». Melvin Charney: Parcours de la réinvention. Dir. Jean-François Chevrier, Johanne Lamoureux et Jun Teshigawara. Caen: FRAC Basse-Normandie, 1998. 118-161.

CHARNEY, Melvin. «Melvin Charney». Entretien avec Jim Donaldson. Alumni Interviews. Montréal: Université McGill, 1999. URL: https://www.mcgill.ca/architecture/alumni/aluminterviews/charney (page consultée le 2 novembre 2020).

CHARNEY, Melvin. Tracking Images : Melvin Charney, Un Dictionnaire... Montréal : Centre canadien d'architecture, 2000.

CHARNEY, Melvin. «Melvin Charney: CEuvres et commentaires». Melvin Charney. Dir. Pierre Landry. Montréal: Musée d'art contemporain de Montréal, 2002. 23-135.

CHARNEY, Melvin et Phyllis LAMBERT. «Une entrevue avec Melvin Charney». In Melvin Charney, Paraboles et autres allégories: l'œuvre de Melvin Charney, 1975-1990. Montréal: Centre canadien d'architecture, 1991. 25-34.

CHARNEY, Melvin et Yasmeen SIDDIQUI. «In Conversation, Yasmeen Siddiqui with Melvin Charney, Montréal, January 4 and 5, $2008 »$. Between Observation and Intervention: The Painted Photographs of Melvin Charney. Dir. Gabriela Rangel et Gwendolyn Owens. New York: Americas Society ; Québec : Musée national des beaux-arts du Québec, 2009. 20-41.

CHÉROUX, Clément, dir. Walker Evans. Paris : Centre Pompidou, 2017.

COHEN, Jean-Louis. «Postface». In Erich Mendelsohn. Amerika. Livre d'images d'un architecte. 1926. Paris : Les Éditions du Demi-Cercle, 1992. 225-241.

HARRIS, David. «La photographie et la fabrication d'images». Melvin Charney. Dir. Pierre Landry. Montréal: Musée d'art contemporain de Montréal, 2002. 13-20.

LANDRY, Pierre, dir. Melvin Charney. Montréal: Musée d'art contemporain de Montréal, 2002.

LE CORBUSIER. «L'espace indicible». L'Architecture d'aujourd'hui (numéro hors-série, 1946) : 9-17.

LEVASSEUR, Élène. Les expériences photographiques formatrices de Melvin Charney: Contours et contributions d'un outil de la pensée en architecture. Thèse de doctorat, Université de Montréal, 2019. 
MARTIN, Louis. «Melvin Charney: A Short Bibliography». On Architecture: Melvin Charney, a Critical Anthology. Dir. Louis Martin. Montréal: McGill-Queen's University Press, 2013. 441-447.

MARTIN, Louis. On Architecture: Melvin Charney, a Critical Anthology. Montréal: McGill-Queen's University Press (McGill-Queen's/Beaverbrook Canadian Foundation Studies in Art History), 2013.

MAXWELL, Robert, dir. Stirling: Writing on Architecture. 1998. Milan: Skira, 2013.

MENDELSOHN, Erich. Amerika. Livre d'images d'un architecte. 1926. Paris: Les Éditions du Demi-Cercle, 1992.

MOHOLY-NAGY, László. Vision in Motion. Chicago : Paul Theobald, 1947.

RUSCHA, Edward. Huit textes, vingt-trois entretiens : 1965-2009. Zurich : JRP Ringier, 2010.

SALOMON, Stephanie et Steve KROETER. «The 1968 Learning from Las Vegas Studio Revisited». Designers \& Books (19 décembre 2013). URL: http://www.designersandbooks.com/blog/1968-learning-las-vegasstudio-revisited (page consultée le 2 novembre 2020).

STIRLING, James. «The Functional Tradition and Expression». Perspecta: The Yale Architectural Journal $6(1960): 88-97$.

VENTURI, Robert et Denise SCOTT BROWN. «A Significance for A\&P Parking Lots or Learning from Las Vegas ». Architectural Forum 128 (mars 1968): 37-43.

VENTURI, Robert, Denise SCOTT BROWN et Steven IZENOUR. Learning from Las Vegas. Cambridge, Massachusetts: The MIT Press, 1972. 
